\title{
Clinical utility of the IHC4 + C score in oestrogen receptor-positive early breast cancer: a prospective decision impact study
}

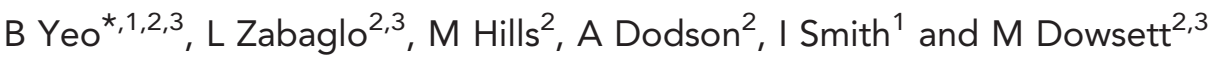 \\ ${ }^{1}$ Department of Medicine, Royal Marsden Hospital, Fulham Road, London SW3 6JJ, UK; ${ }^{2}$ Academic Department of \\ Biochemistry, Royal Marsden Hospital, Fulham Road, London SW3 6JJ, UK and ${ }^{3}$ Institute of Cancer Research, Fulham Road, \\ London SW3 6JB, UK
}

Background: Most oestrogen receptor (ER)-positive early breast cancer diagnosed today is highly curable with multimodality treatment. Systemic adjuvant treatments including endocrine therapy and chemotherapy have made a significant contribution to the increasing cure rates over the past three decades. However not all women will require chemotherapy. The IHC4 $+\mathrm{C}$ score is a prognostic tool that integrates four immunohistochemical measures with clinicopathological features to estimate the residual risk of distant recurrence at 10 years in post-menopausal women with ER-positive breast cancer who have received 5 years of endocrine therapy. Retrospective studies indicate that the test can identify a set of women that are at such low risk of recurrence that chemotherapy can be of little benefit.

Methods: In this study, 124 patients were prospectively selected from the multidisciplinary team meeting between January 2013 and April 2014 for IHC4 + C testing. Adjuvant systemic treatment recommendations by clinicians were recorded without and with the availability of the score in addition to the patient's decision.

Results: There was concordance in the MDT's recommendation without and with the availability of the score in $73 \%$ of cases. Clinicians recommended chemotherapy or at least its discussion to $74(59 \%)$ patients, which fell to 32 (34\%) patients after the IHC4 + C score was made available, sparing one in four tested patients a chemotherapy recommendation, along with its toxicity and expense.

Conclusion: This decision impact study shows that when used by clinicians in the multidisciplinary team meeting for adjuvant decision-making, a significant proportion of patients are spared chemotherapy recommendations.

Adjuvant systemic therapy has made a significant contribution to the improved outcomes for patients diagnosed with oestrogen receptor (ER)-positive early breast cancer over the past three decades. Five years of adjuvant tamoxifen therapy has been estimated to improve breast cancer mortality by about a third (Early Breast Cancer Trialists Collaborative G, 2005) with superior benefit seen with the use of aromatase inhibitors in postmenopausal women (Breast International Group 1-98 Collaborative $\mathrm{G}$ et al, 2005; Howell et al, 2005; Cuzick et al, 2010). More recently, extending hormonal therapy beyond 5 to 10 years has shown further benefit using either tamoxifen (Davies et al, 2013;
Gray et al, 2013) or 5 years of an aromatase inhibitor after an initial 5 years of tamoxifen (Goss et al, 2007).

Polychemotherapy provides a further reduction in breast cancer mortality when given in addition to endocrine therapy, improving breast cancer mortality on average by a third (RR $0 \cdot 78$, SE $0 \cdot 07$, $2 \mathrm{p}=0 \cdot 001$ in women 55-69 years) (Early Breast Cancer Trialists' Collaborative $G$ et al, 2012). However its use is associated with potential toxicities both in the short term such as alopecia, nausea, vomiting and neutropenic sepsis and in the longer term rarer effects such as infertility, cardiac impairment and haematological malignancies. 
Not all women with ER-positive disease will have substantial gains from chemotherapy; this includes those with such a good prognosis, taking into consideration their endocrine therapy that they could not benefit meaningfully from additional treatment. It would be beneficial for such patients to be spared these toxicities. Traditionally tumour size, grade and nodal burden have dominated decision-making, particularly through the use of decision aids such as the Nottingham prognostic index and Adjuvant! Online (AoL). However a number of prognostic indices have been developed that improve prognostic accuracy.

The IHC4 + C score is a prognostic tool that estimates the residual risk of distant recurrence at 10 years in post-menopausal women with ER-positive breast cancer who have received 5 years of endocrine therapy. It incorporates both immunohistochemical parameters of ER, progesterone receptor (PgR), HER2 and Ki67 as well as the clinicopathological parameters of tumour size, grade, nodal status and type of endocrine therapy administered for 5 years (tamoxifen $v s$ aromatase inhibitor). It was developed from a retrospective analysis of 1125 patients with ER-positive disease from the TransATAC cohort who did not received chemotherapy, validated in an independent cohort of 786 patients and has been shown to perform similarly to the Genomic Health recurrence score (RS) in predicting distant recurrence (Cuzick et al, 2011). In this journal, Stephen et al, 2014 showed that in an endocrine treated population from the TEAM trial, the IHC4 provided stronger prognostic information when compared with the Mammostrat score.

When used in adjuvant decision-making, AoL, RS and IHC4 + C, categorise patients into low-, intermediate- and high-risk categories. Patients in the low-risk range are usually recommended endocrine therapy alone, and those in the high-risk range are offered chemotherapy in addition to endocrine therapy. There is no consensus as to the optimal treatment for those in the intermediate-risk range and while clinical trials are currently underway to address this uncertainty (MINDACT and TAILORX), many patients are currently offered chemotherapy. In a retrospective analysis of 101 patients who had both AoL and IHC4 + C risk estimates calculated, the $\mathrm{IHC} 4+\mathrm{C}$ score reclassified more than half of the patients stratified as being of intermediate risk using AoL to low risk (Barton et al, 2012).

The need to study the clinical utility of the IHC4 $+\mathrm{C}$ score has recently been identified by the National Institute for Health and Care Excellence (NICE) in its 2013 guidance paper on gene expression profiling and expanded immunohistochemistry tests for guiding adjuvant chemotherapy decisions in early breast cancer management (NICE diagnostic guidance 10, 2013). The aim of this prospective study was to determine the effect of the $\mathrm{IHC} 4+\mathrm{C}$ score on adjuvant systemic decision-making in the setting of the multidisciplinary team meeting (MDT) at our institution, the forum for making adjuvant treatment recommendations. The MDT has been shown to provide consistent and evidence-based decision-making and is now part of the standard of care in the management of breast cancer (Saini et al, 2012).

\section{PATIENTS AND METHODS}

Patient population. Post-operative ER-positive breast cancer patients were prospectively selected for IHC4 $+\mathrm{C}$ testing during the breast unit MDT at The Royal Marsden Hospital between January 2013 and April 2014 (Figure 1). Patients were eligible if they were 50 years or older at the time of diagnosis with an ERpositive, HER2-negative (HER2 0 or $1+$ on immunohistochemistry or DDISH-negative if HER2 $2+$ ) breast tumour with pathological lymph node stage N0/1. All patients were treated with primary surgery. Nodal involvement with micrometastases and/or ITCs was classified as node-negative for the purpose of scoring. Patients with T4 disease, incomplete axillary staging, before neoadjuvant therapy and patients in whom the adjuvant decision would be in no way changed by the availability of the score (e.g., significant co-morbidities, patient's known intention against chemotherapy), were excluded.

Study design. Adjuvant treatment decisions were recorded at three time points during this study (Figure 1). The initial recommendation by the MDT clinicians for adjuvant systemic therapy was recorded (Decision 1). The IHC4 + C score was requested by clinicians to be derived only for patients in whom it was felt the IHC4 $+\mathrm{C}$ could affect decision-making. The IHC4 staining was requested and the IHC4 $+\mathrm{C}$ prediction of the risk of distant recurrence at 10 years was presented to the MDT 1 week later. Their subsequent recommendation with information provided from the score was recorded (Decision 2). The patient's final decision after discussion in the clinic as to their accepted choice of adjuvant therapy was also recorded (Decision 3 ).

This decision was reported in one of three ways: (i) for endocrine therapy alone (no chemotherapy recommended); (ii) for chemotherapy (in addition to endocrine therapy); or (iii) for a discussion of chemotherapy (i.e., a less definitive indication for chemotherapy but that it should be discussed with the patient; that discussion then could lead to the patient accepting chemotherapy). The study was approved as a service evaluation by the institution's clinical audit committee.

Study endpoints. The primary endpoint was the proportion of patients who had a change in their adjuvant systemic treatment recommendation by the MDT due to the availability of the score. Secondary goals were to correlate the IHC4 + C score with the AoL score in predicting risk of recurrence with endocrine therapy and to calculate the proportion of patients who have a change in their actual choice of adjuvant treatment (Decision 3) from the MDT recommended treatment (Decision 2).

The IHC4 + C score calculation. Clinicopathological data was collected prospectively from the MDT including age and menopausal status and type of endocrine therapy intended. Immunohistochemistry for ER, PgR, HER2 and Ki67 was performed as previously described (Dowsett et al, 2008). ER was quantified by using the $\mathrm{H}$-score, which is defined as the percentage of cell staining weakly plus $2 \times$ the percentage of cell staining moderately, plus $3 \times$ the percentage of cell staining strongly, and was considered positive if $>1$. The variable $\mathrm{ER}_{10}$ was derived by dividing the $\mathrm{H}$-score by 30 to obtain a variable with a range of $0-10$. PgR was scored as the percentage of cells staining positive, and was divided by 10 to obtain a variable between 0 and 10 (PgR10). HER2 status was assessed as either positive $(3+$ or $2+$ and CISH or FISH positive) or negative $(0 / 1+$ or $2+$ and CISH or FISH negative). Ki-67 scores were recorded as the percentage of positively staining cancer cells using the MIB1 antibody, in five representative high-power fields (Zabaglo et al, 2010).

The IHC4 $+\mathrm{C}$ score was calculated using the addition of two algorithms:

(i) The IHC4 score

$\mathrm{IHC} 4=94.7 \times\left\{-0.100 \mathrm{ER}_{10}-0.079 \mathrm{PgR}_{10}+0.586 \mathrm{HER} 2+0.240 \ln (1+4 \mathrm{xKi} 67)\right\}$

using an adjustment for Ki67 as previously described because of the differences in scoring from manual methods (Barton et al, 2012).

(ii) The clinical score $(C)$

$$
\begin{aligned}
C= & 100 \times\left\{0.417 \mathrm{~N}_{1-3}+1.566 \mathrm{~N}_{>3}++0.930\left(0.497 \mathrm{~T}_{1-2}+0.882 \mathrm{~T}_{3}\right.\right. \\
& \left.\left.+1.838 \mathrm{~T}_{>3}+0.559 \mathrm{Gr}_{2}+0.970 \mathrm{Gr}_{3}+0.130 \mathrm{Age}_{\geq 65}-0.149 \mathrm{Ana}\right)\right\}
\end{aligned}
$$

with N, T, Gr and Age representing categories of nodal status, tumour size, grade and patient age, respectively, and Ana 


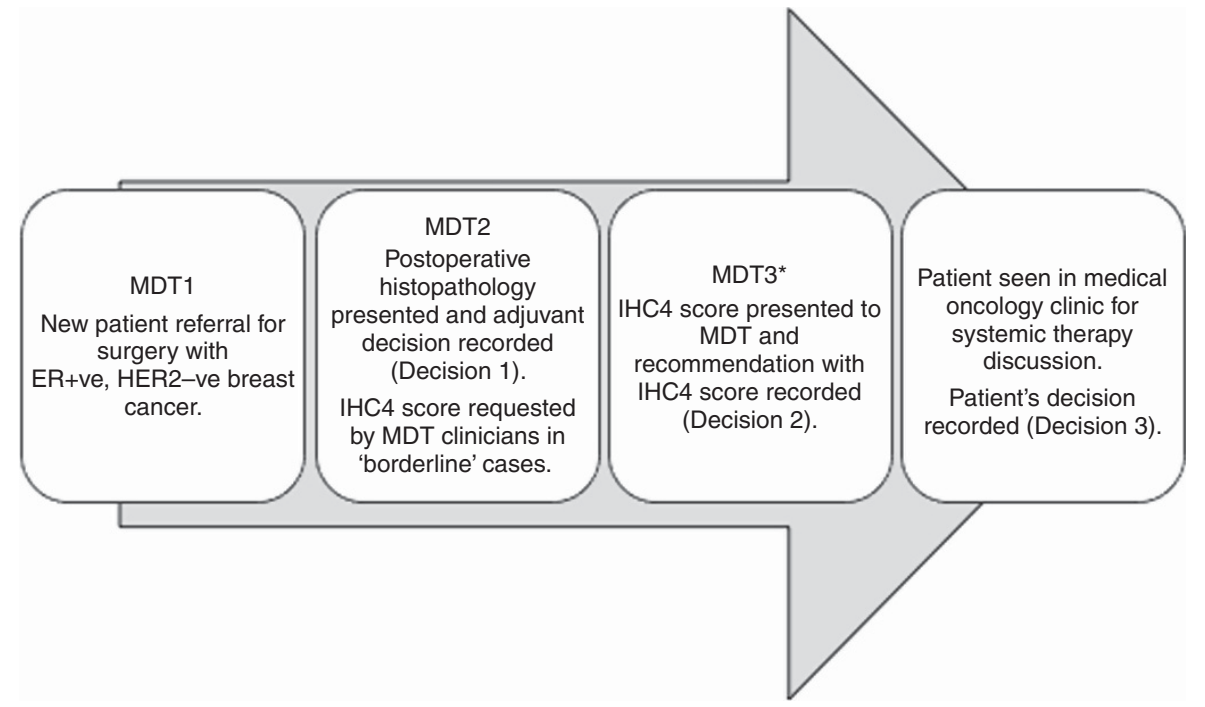

Figure 1. Patient decision pathway. ${ }^{\star}$ Time between MDT2 and MDT3 1 week.

representing treatment with anastrozole as opposed to tamoxifen (Cuzick et al, 2010; Barton et al, 2012).

AoL distant recurrence calculation. An adjustment to AoL was required in order to compare the estimated residual risk of distant recurrence at 10 years between $\mathrm{AoL}$ and $\mathrm{IHC} 4+\mathrm{C}$. As previously described (Barton et al, 2012) the AoL residual risk of breast cancer recurrence was calculated using the AoL's estimate of breast cancer mortality at 10 years and subtracting the estimated benefit of 5 years of endocrine therapy, leaving the 10-year breast cancerspecific mortality. This was then multiplied by 1.25 , to account for only the distant recurrence events (estimated to be $25 \%$ greater than the breast cancer mortality events in the ATAC study at 10 years (Cuzick et al, 2010)).

Statistical analysis. The risk cutoffs used of 10 and 20\% residual risk of distant recurrence at 10 years were chosen to correspond with the 18 and 30 RS risk of distant recurrence at 10 years that has previously been is used to classify patients as being at intermediate risk (Howell et al, 2005; Dowsett et al, 2010; Barton et al, 2012). The planned sample size of 100 patients at this single institution was previously sufficient to demonstrate the reclassification by $\mathrm{IHC} 4+\mathrm{C}$ of patients into a lower risk group (Barton et al, 2012).

The relationship between AoL and IHC4 $+\mathrm{C}$ was assessed on a continuous basis graphically and by Spearman's Rank correlation. The agreement of the AoL and IHC4 $+\mathrm{C}$ on a categorical basis was tested using $\kappa$ statistic where a $\kappa$ statistic $<0.4$ is considered poor agreement, $0.4-0.75$ is considered moderate agreement and $>0.75$ is considered a strong agreement.

\section{RESULTS}

Patient characteristics. Between January 2013 and April 2014, 124 patients met eligibility criteria for the study with tissue suitable for IHC4 $+\mathrm{C}$ assessment. Clinical and tumour characteristics are summarised in Table 1 . The median patient age was 59 years, the median tumour size was $20 \mathrm{~mm}$ and most were grade $2(65 \%)$. Twenty-six percent of patients had macroscopic lymph node involvement and most (85\%) patients had an aromatase inhibitor as their recommended endocrine therapy leaving 19 patients (15\%) recommended for tamoxifen, predominantly because of perimenopausal status.

\section{Table 1. Clinicopathological characteristics}

\begin{tabular}{|c|c|}
\hline Factor & No. of patients \\
\hline \multicolumn{2}{|l|}{ Age } \\
\hline $50-59$ & $66(53 \%)$ \\
\hline $60-69$ & $46(37 \%)$ \\
\hline 70-79 & $12(10 \%)$ \\
\hline \multicolumn{2}{|l|}{ Tumour size (mm) } \\
\hline$<20$ & 48 (39\%) \\
\hline $20-29$ & 48 (39\%) \\
\hline $30-49$ & $24(19 \%)$ \\
\hline$\geqslant 50$ & $4(3 \%)$ \\
\hline \multicolumn{2}{|l|}{ Histological subtype } \\
\hline IDC & $100(81 \%)$ \\
\hline ILC & $16(13 \%)$ \\
\hline mixed IDC/ILC & $6(5 \%)$ \\
\hline mucinous & $2(1 \%)$ \\
\hline \multicolumn{2}{|l|}{ Grade } \\
\hline 1 & $17(14 \%)$ \\
\hline 2 & $81(65 \%)$ \\
\hline 3 & $26(21 \%)$ \\
\hline \multicolumn{2}{|l|}{ LVI } \\
\hline Yes & $32(26 \%)$ \\
\hline No & 92 (74\%) \\
\hline \multicolumn{2}{|l|}{ Nodal stage } \\
\hline N0 (node negative ${ }^{a}$ ) & $92(74 \%)$ \\
\hline N1 (1-3 nodes positive) & $32(26 \%)$ \\
\hline \multicolumn{2}{|l|}{ ER status (H-score) } \\
\hline 10-99 & $2(2 \%)$ \\
\hline 100-199 & $63(51 \%)$ \\
\hline$\geqslant 200$ & 59 (47\%) \\
\hline \multicolumn{2}{|l|}{ PgR status (\%) } \\
\hline$<5$ & $16(13 \%)$ \\
\hline $5-39$ & $16(13 \%)$ \\
\hline $40-79$ & $26(21 \%)$ \\
\hline$\geqslant 80$ & $66(53 \%)$ \\
\hline \multicolumn{2}{|l|}{ Ki67\% } \\
\hline$<10$ & $35(28 \%)$ \\
\hline 10-19 & 38 (31\%) \\
\hline$\geqslant 20$ & 51 (41\%) \\
\hline
\end{tabular}




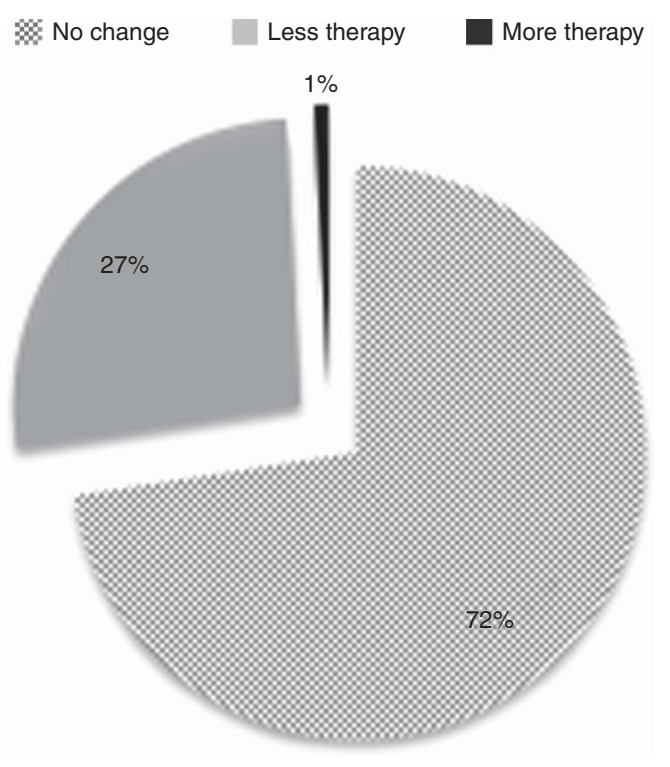

Figure 2. Concordant and disconcordant MDT decisions between Decisions 1 and 2.

Comparison between MDT decisions with and without the availability of IHC4 + C score (Decisions $\mathbf{1}$ and 2). The IHC4+ $\mathrm{C}$ score led to more endocrine therapy alone recommendations being made by the MDT. Figure 2 shows the concordant and discordant decisions with $\mathrm{IHC} 4+\mathrm{C}$ scoring. There was concordance in the MDT's recommendation between Decisions 1 and 2 in 90 cases (72\%). In the 34 discordant cases, all but one led to endocrine therapy alone being recommended rather than chemotherapy plus endocrine therapy after the $\mathrm{IHC} 4+\mathrm{C}$ score was given to the MDT. In Table 2: from Decision 1, 50 (40\%) patients had an endocrine therapy alone recommendation and this increased to 82 (66\%) patients at Decision 2. There were no 'discuss chemotherapy' recommendations at Decision 2.

Figure 3 shows individual changes in treatment recommendations for all patients at Decisions 1,2 and 3. At Decision 1, chemotherapy or at least its discussion was recommended in 74 (59\%) patients. This fell to $42(34 \%)$ at Decision 2, potentially sparing $32(26 \%)$ patients a chemotherapy recommendation. In the 32 patients with node-positive disease (not including micrometastases), a subgroup traditionally thought to be of increased risk of relapse, 11 (34\%) patients had their initial chemotherapy recommendation changed to endocrine therapy alone.

Comparison between MDT recommendation to patient and patient's decision (Decisions 2 and 3). Patients adhered to the recommendation by the clinician $82 \%$ of the time (102 out of 124 ) of cases (Figure 3). Fifteen patients who were recommended chemotherapy in Decision 2, chose not to have it at Decision 3. Five patients who were recommended endocrine therapy alone in Decision 2 chose to have chemotherapy at Decision 3. Two patients who were recommended endocrine therapy at Decision 2 chose to have no adjuvant therapy in Decision 3.

Comparison between IHC4 + C score and AOL. Figure 4 shows the relationship between AoL and IHC4 $+\mathrm{C}$ estimates of risk of distant recurrence at 10 years for the 124 patients. The Spearman's Rank correlation was 0.73. Agreement between the categories created for the IHC4 $+\mathrm{C}$ score and the AoL score was $54 \%$ (CI $45.7-63.8 \% ; \kappa=0.32$ ). AoL generally estimated patients at higher risk than IHC4 + C. AoL estimated $31 \%$ of patients as high risk, while $\mathrm{IHC} 4+\mathrm{C}$ rated only $10 \%$ patients as high risk. From the 38 patients classified as high risk by AoL,
Table 2. Change in MDT decision-making without (Decision 1) and with (Decision 2) the IHC4 + C score

\begin{tabular}{|l|c|c|}
\hline & Decision 1 & Decision 2 \\
\hline Endocrine therapy & $50(40 \%)$ & $82(66 \%)$ \\
\hline Discuss chemotherapy & $45(36 \%)$ & 0 \\
\hline Recommend chemotherapy & $29(23 \%)$ & $42(34 \%)$ \\
\hline
\end{tabular}

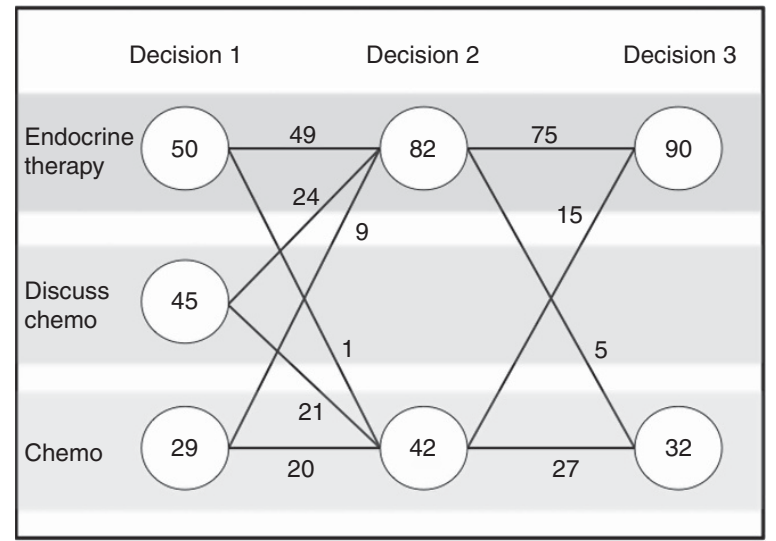

Figure 3. Adjuvant systemic therapy treatment decisions. Fifteen patients who were recommended chemotherapy in Decision 2, chose not to have it at Decision 3. Five patients who were recommended endocrine therapy alone in Decision 2 chose to have chemotherapy at Decision 3. Two patients who were recommended endocrine therapy at Decision 2 chose to have no adjuvant therapy in Decision 3.

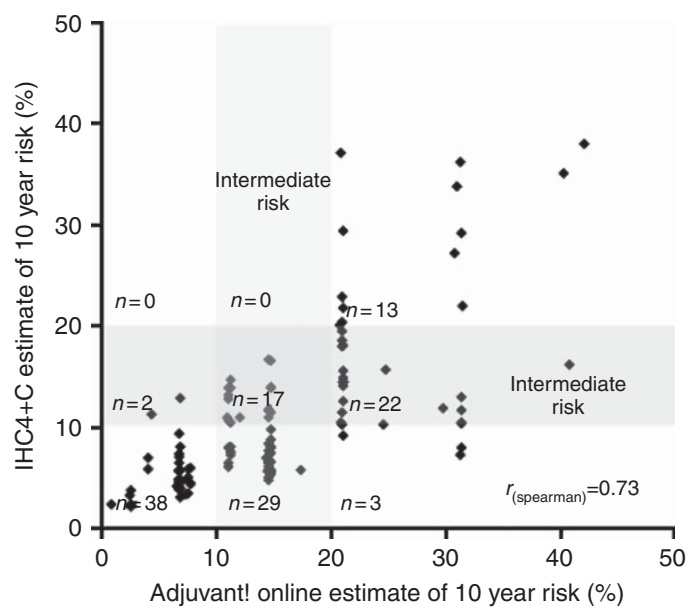

Figure 4. Spearman's rank correlation between AoL and estimates of risk of distant recurrence at 10 years. Agreement: $54.8 \%(\mathrm{Cl} 45.7-$ $63.8 \% ; \kappa=0.32$.

$58 \%$ were reclassified as intermediate risk by IHC4 and another $8 \%$ reclassified in the low-risk range. From the 46 patients in the intermediate AoL range, $63 \%$ were reclassified into the low-risk range using $\mathrm{IHC} 4+\mathrm{C}$. IHC4 $+\mathrm{C}$ rated $56 \%$ of patients as low risk, as opposed to AoL which rated only $30 \%$ as low risk. Only two patients were reclassified from low-tointermediate risk by the use of $\mathrm{IHC} 4+\mathrm{C}$ score, and none were reclassified to high risk.

\section{DISCUSSION}

To our knowledge, this is the first prospective study of the clinical utility of the IHC4 $+\mathrm{C}$ score. The population was clinically 
relevant: all patients in whom the score was performed were felt by the MDT to have either a minimal or low benefit from chemotherapy, such that additional information provided by the score may help to reinforce their initial recommendation of endocrine therapy alone, or change their initial recommendation from chemotherapy to endocrine therapy alone. Over the 15months period of recruitment for this study, $\sim 850$ women over 50 years of age with ER-positive breast cancer were presented at the post-operative MDT for decision-making, leading to 124 (15\%) patients having had an IHC4 $+\mathrm{C}$ test requested by clinicians. For patients in whom it was felt the availability of the score could not have any impact on decision-making, it was not requested (e.g., co-morbidities would preclude chemotherapy anyway). Long-term follow-up in these patients is planned.

The IHC4 + C score provided useful information to the MDT in two ways.

1. Reducing chemotherapy recommendations in a borderline population of patients. From those patients in whom the initial recommendation was either for a discussion or clear recommendation of chemotherapy, $26 \%$ of these patients were subsequently recommended endocrine therapy alone, as guided by the residual recurrence estimates provided by the score, and hence sparing a significant proportion of women the potential short and long-term toxicities of chemotherapy, as well as sparing health-care providers the costs in providing such treatment. It is estimated that in the United Kingdom the cost of six cycles of second generation chemotherapy with fluorouracil, epirubicin and cyclophosphamide is $\sim £ 3500$ (Campbell et al, 2011). In our series, 32 patients were potentially spared chemotherapy, amounting to a direct chemotherapy cost saving of $£ 112000$ over a period of 15 months of patient recruitment, not withstanding the cost of hospital admissions for the treatment of febrile neutropenia as well as sparing of undesirable side effects of chemotherapy for patients.

2. Providing reassurance in concordant cases. Patients in this study were requested for an $\mathrm{IHC} 4+\mathrm{C}$ on the basis that the clinician felt that more information provided by the score could assist in their decision-making. Most patients (73\%) had concordant decisions recommended before and after scoring. We estimated that the MDT considered their initial recommendation for treatment to be 'borderline' for 47 out of these 91 concordant cases. Hence concordant scoring helped to confirm their decision and provide reassurance to the clinician.

The IHC4 + C score is a prognostic tool and does not offer predictive information. In our study, the use of this prognostic tool enabled a large proportion of patients to be treated with endocrine therapy alone. As shown in Figure 3, the initial MDT recommendation confidently spared chemotherapy in 50 (40\%) patients (Decision 1). With the availability of the score 90 (72\%) patients ultimately decided on endocrine therapy as their treatment of choice (Decision 3).

One criticism of this study may be that the benefit of the IHC4 + C score in reducing chemotherapy recommendations may be overestimated by the group in whom a discussion of chemotherapy was recommended. In 45 out of the 124 (36\%) patients in whom an IHC4 + C score was requested by the MDT, the original recommendation was for a discussion regarding chemotherapy. With the availability of the score, 21 of these were still recommended chemotherapy, leaving 24 patients to be recommended endocrine therapy only. Without the availability of the IHC4 + C score to the MDT, there can be only an estimation of how many of these patients who were recommended for a discussion would have actually had chemotherapy. Using our best clinical estimate and based on prior experience, in the absence of the IHC4 $+\mathrm{C}$ score, chemotherapy would most likely have been given in $36(80 \%)$ patients in whom a discussion of chemotherapy was the initial recommendation. This estimate is based on the fact that in the 'discuss chemotherapy' cohort, 13 cases were $>3 \mathrm{~cm}$; an additional 12 were grade 3 and an additional 11 patients who were not grade 3 or over $3 \mathrm{~cm}$ had nodal involvement. It was felt that it would be inappropriate to ask patients, in what is often a stressful time at the point of deciding on chemotherapy or not, what their decision would be without and with the availability of the IHC4+ C score.

The IHC4+C score is currently only validated in the post-menopausal patient. However it may be seen that the information provided by the score would be equally if not more usefully applied to premenopausal women, particularly given the potential effects chemotherapy may have on fertility in this cohort. This would require testing the calculator in a large set of premenopausal women, treated with tamoxifen but not chemotherapy

In this prospective single institution study, we have shown that the IHC4 $+\mathrm{C}$ score can be performed in real time, making it available to the MDT for decision-making. The IHC4 + C score was made available to the MDT within 1 week and in time for medical oncology appointments where most adjuvant systemic therapy is discussed. Scoring requires a dedicated breast pathologist, and scoring of immunohistochemical markers provided routinely by most service histopathology laboratories in the United Kingdom. Clinical information using the patient age and intended endocrine therapy (aromatase inhibitor $v s$ tamoxifen) are included in the calculator. It is important to note that this information should be gathered on pretreatment samples (i.e., no neoadjuvant therapy or other presurgical treatment administered).

Commercially available molecular signatures (Oncotype DX (Genomic Health, Redwood City, CA, USA); Endopredict (Sividon Diagnostics, Cologne, Germany); Prosigna (Nanostring, Seattle, WA, USA)), remain inaccessible to the majority of NHS patients at this time. Oncotype DX may be available through an access scheme for some eligible NHS patients with early stage hormone-receptor positive, HER2-negative breast cancer who are considered to be at intermediate risk (NICE diagnostic guidance 10, 2013). The IHC4 + C score has the potential to fulfil these unmet needs by providing similar prognostic information to assist in adjuvant decision-making without the need to send tissue externally, and without the significant financial cost, to the patient, insurer or trust for doing so. The cost of performing the score is estimated to be less than $£ 200$ per case at our institution.

All scoring was performed within our single institution by our research team, which has extensive experience in conducting the tests and assessing the results in order to produce standardised input for the IHC4 algorithm and this may be seen as a limitation of the study. If IHC4 $+\mathrm{C}$ scoring is to be routinely used across the $\mathrm{UK}$, there will be a need to validate local procedures and to quality assure performance on a continuing basis. The standardisation of Ki67 assessment in particular has been shown to be problematic (Dowsett et al, 2011). However there is evidence, at least from the UK, that Ki67 immunohistochemistry is technically reproducible when laboratories validate pre-analytical and technical aspects appropriately and participate in an external quality assessment scheme (Parry et al, 2014), and efforts are ongoing by an international study group to improve its analytical reproducibility (Polley et al, 2013). We have recently established that the IHC4 score is tolerant of multiple different IHC platforms (Dodson et al, 2015). Nonetheless it is important that labs should establish that their own methodology yields acceptable concordance with reference methodology (Cuzick et al, 2011) before implementation. Ultimately we envisage that the IHC4 + C calculator may be universally available in an online 
format like AoL where the individual components can be entered by the treating team.

\section{ACKNOWLEDGEMENTS}

We acknowledge the support of The Cridlan Trust, Breakthrough Breast Cancer and The Royal Marsden NIHR Biomedical Research Centre.

\section{REFERENCES}

Barton S, Zabaglo L, A'Hern R, Turner N, Ferguson T, O’Neill S, Hills M, Smith I, Dowsett M (2012) Assessment of the contribution of the $\mathrm{IHC} 4+\mathrm{C}$ score to decision making in clinical practice in early breast cancer. Br J Cancer 106(11): 1760-1765.

Thurlimann B, Keshaviah A, Coates AS, Mouridsen H, Mauriac L, Forbes JF, Paridaens R, Castiglione-Gertsch M, Gelber RD, Rabaglio M, Smith I, Wardley A, Price KN, Goldhirsch A. Breast International Group 1-98 Collaborative G (2005) A comparison of letrozole and tamoxifen in postmenopausal women with early breast cancer. $N$ Engl J Med 353(26): 2747-2757.

Campbell HE, Epstein D, Bloomfield D, Griffin S, Manca A, Yarnold J, Bliss J, Johnson L, Earl H, Poole C, Hiller L, Dunn J, Hopwood P, Barrett-Lee P, Ellis P, Cameron D, Harris AL, Gray AM, Sculpher MJ (2011) The costeffectiveness of adjuvant chemotherapy for early breast cancer: a comparison of no chemotherapy and first, second, and third generation regimens for patients with differing prognoses. Eur J Cancer 47(17): 2517-2530.

Cuzick J, Dowsett M, Pineda S, Wale C, Salter J, Quinn E, Zabaglo L, Mallon E, Green AR, Ellis IO, Howell A, Buzdar AU, Forbes JF (2011) Prognostic value of a combined estrogen receptor, progesterone receptor, Ki-67, and human epidermal growth factor receptor 2 immunohistochemical score and comparison with the Genomic Health recurrence score in early breast cancer. J Clin Oncol 29(32): 4273-4278.

Cuzick J, Sestak I, Baum M, Buzdar A, Howell A, Dowsett M, Forbes JF. Investigators AL (2010) Effect of anastrozole and tamoxifen as adjuvant treatment for early-stage breast cancer: 10-year analysis of the ATAC trial. Lancet Oncol 11(12): 1135-1141.

Davies C, Pan H, Godwin J, Gray R, Arriagada R, Raina V, Abraham M, Medeiros Alencar VH, Badran A, Bonfill X, Bradbury J, Clarke M, Collins R, Davis SR, Delmestri A, Forbes JF, Haddad P, Hou MF, Inbar M, Khaled H, Kielanowska J, Kwan WH, Mathew BS, Mittra I, Muller B, Nicolucci A, Peralta O, Pernas F, Petruzelka L, Pienkowski T, Radhika R, Rajan B, Rubach MT, Tort S, Urrutia G, Valentini M, Wang Y, Peto R. Adjuvant Tamoxifen: Longer Against Shorter Collaborative Group (2013) Longterm effects of continuing adjuvant tamoxifen to 10 years versus stopping at 5 years after diagnosis of oestrogen receptor-positive breast cancer: ATLAS, a randomised trial. Lancet 381(9869): 805-816.

Dodson A, Zabaglo L, Yeo B, Miller K, Smith I, Dowsett M (2015) Abstract P5-10-06: Risk of recurrence estimates with IHC4 are tolerant of variations in staining and scoring. Cancer Res 75(9 Supplement): P5-10.

Dowsett M, Allred C, Knox J, Quinn E, Salter J, Wale C, Cuzick J, Houghton J, Williams N, Mallon E, Bishop H, Ellis I, Larsimont D, Sasano H, Carder P, Cussac AL, Knox F, Speirs V, Forbes J, Buzdar A (2008) Relationship between quantitative estrogen and progesterone receptor expression and human epidermal growth factor receptor 2 (HER-2) status with recurrence in the Arimidex, Tamoxifen, Alone or in Combination trial. J Clin Oncol 26(7): 1059-1065.

Dowsett M, Cuzick J, Wale C, Forbes J, Mallon EA, Salter J, Quinn E, Dunbier A, Baum M, Buzdar A, Howell A, Bugarini R, Baehner FL, Shak S (2010) Prediction of risk of distant recurrence using the 21-gene recurrence score in node-negative and node-positive postmenopausal patients with breast cancer treated with anastrozole or tamoxifen: a TransATAC study. J Clin Oncol 28(11): 1829-1834.

Dowsett M, Nielsen TO, A'Hern R, Bartlett J, Coombes RC, Cuzick J, Ellis M, Henry NL, Hugh JC, Lively T, McShane L, Paik S, Penault-Llorca F, Prudkin L, Regan M, Salter J, Sotiriou C, Smith IE, Viale G, Zujewski JA,
Hayes DF (2011) Assessment of Ki67 in breast cancer: recommendations from the International Ki67 in Breast Cancer working group. J Natl Cancer Inst 103(22): 1656-1664.

Early Breast Cancer Trialists' Collaborative G (2005) Effects of chemotherapy and hormonal therapy for early breast cancer on recurrence and 15-year survival: an overview of the randomised trials. Lancet 365(9472): 1687-1717.

Early Breast Cancer Trialists' Collaborative GPeto R, Davies C, Godwin J, Gray R, Pan HC, Clarke M, Cutter D, Darby S, McGale P, Taylor C, Wang YC, Bergh J, Di Leo A, Albain K, Swain S, Piccart M, Pritchard K (2012) Comparisons between different polychemotherapy regimens for early breast cancer: meta-analyses of long-term outcome among 100,000 women in 123 randomised trials. Lancet 379(9814): 432-444.

Goss PE, Ingle JN, Martino S, Robert NJ, Muss HB, Piccart MJ, Castiglione M, Tu D, Shepherd LE, Pritchard KI, Livingston RB, Davidson NE, Norton L, Perez EA, Abrams JS, Cameron DA, Palmer MJ, Pater JL. National Cancer Institute of Canada Clinical Trials Group MA (2007) Efficacy of letrozole extended adjuvant therapy according to estrogen receptor and progesterone receptor status of the primary tumor: National Cancer Institute of Canada Clinical Trials Group MA.17. J Clin Oncol 25(15): 2006-2011.

Gray RG, Rea D, Handley K, Bowden SJ, Perry P, Earl HM, Poole CJ, Bates T, Chetiyawardana S, Dewar JA, Fernando IN, Grieve R, Nicoll J, Rayter Z, Robinson A, Salman A, Yarnold J, Bathers S, Marshall A, Lee M (2013) aTTom: Long-term effects of continuing adjuvant tamoxifen to 10 years versus stopping at 5 years in 6,953 women with early breast cancer. In ASCO Annual Meeting Proceedings, vol 31, No 18_Suppl, p 5.

Howell A, Cuzick J, Baum M, Buzdar A, Dowsett M, Forbes JF, Hoctin-Boes G, Houghton J, Locker GY, Tobias JS. Group AT (2005) Results of the ATAC (Arimidex, Tamoxifen, Alone or in Combination) trial after completion of 5 years' adjuvant treatment for breast cancer. Lancet 365(9453): 60-62.

NICE diagnostic guidance 10 (2013) Gene expression profiling and expanded immunohistochemistry tests for guiding adjuvant chemotherapy decisions in early breast cancer management: MammaPrint OD, IHC4 and Mammostrat.

Parry S, Steele D, Miller K, Ibrahim M (2014) Variability in Ki-67 Breast Carcinoma Immunohistochemistry (IHC) Staining Shown by UK External Quality Assessments. 26 $6^{\text {th }}$ European Congress of Pathology; 30 August-3 September 2014; London, UK. Poster PS-03-138.

Polley MY, Leung SC, McShane LM, Gao D, Hugh JC, Mastropasqua MG, Viale G, Zabaglo LA, Penault-Llorca F, Bartlett JM, Gown AM, Symmans WF, Piper T, Mehl E, Enos RA, Hayes DF, Dowsett M, Nielsen TO. International Ki67 in Breast Cancer Working Group of the Breast International G, North American Breast Cancer G (2013) An international Ki67 reproducibility study. J Natl Cancer Inst 105(24): 1897-1906.

Saini KS, Taylor C, Ramirez AJ, Palmieri C, Gunnarsson U, Schmoll HJ, Dolci SM, Ghenne C, Metzger-Filho O, Skrzypski M, Paesmans M, Ameye L, Piccart-Gebhart MJ, de Azambuja E (2012) Role of the multidisciplinary team in breast cancer management: results from a large international survey involving 39 countries. Ann Oncol 23(4): 853-859.

Stephen J, Murray G, Cameron DA, Thomas J, Kunkler IH, Jack W, Kerr GR, Piper T, Brookes CL, Rea DW, van de Velde CJ, Hasenburg A, Markopoulos C, Dirix L, Seynaeve C, Bartlett JM (2014) Time dependence of biomarkers: non-proportional effects of immunohistochemical panels predicting relapse risk in early breast cancer. Br J Cancer 111(12): 2242-2247.

Zabaglo L, Salter J, Anderson H, Quinn E, Hills M, Detre S, A'Hern R, Dowsett M (2010) Comparative validation of the SP6 antibody to Ki67 in breast cancer. J Clin Pathol 63(9): 800-804.

This work is published under the standard license to publish agreement. After 12 months the work will become freely available and the license terms will switch to a Creative Commons AttributionNonCommercial-Share Alike 4.0 Unported License. 